\title{
GNSS Real Time Performance Monitoring and CNS/ATM Implementation
}

\author{
Karen Van Dyke, Jon Parmet, Christopher Dufresne, Andrew Hansen \\ DOT/RITA/Volpe Center \\ Richard Cole, Jennifer Campbell, Jianming She, Yi Bian, Robert Loh \\ Innovative Solutions International
}

\section{ABSTRACT}

The global transition to communications, navigation, surveillance / air traffic management (CNS/ATM) technology is moving forward at an increasing pace. A critical part of the CNS/ATM concept is the ability to monitor, analyze, and distribute aeronautical information pertaining to system performance. The ability to relay real-time system performance to the user community is required in order to develop and distribute Notices to Airmen (NOTAMs), manage traffic flow, and predict service level availability.

Brazil's Department of Air Space Control (DECEA) in cooperation with the US Volpe Transportation Center and Innovative Solutions International (ISI), Inc., has embarked on a program to develop and deploy the first real-time GNSS performance monitoring capability for operational use. The system will be able to monitor the operational status of a GNSS system in real time, as well as predict its future performance as well as possible. This paper describes the functions of the GNSS Performance Monitoring System (GPMS).

\section{INTRODUCTION}

Satellite navigation will ultimately become the primary means of navigation service in many areas of the world. In addition, the scope of satellite-based navigation will extend beyond air navigation, and will eventually have a profound effect on the everyday lives of ordinary citizens. Therefore, the capability to integrate, monitor, and assess the operational performance of these systems is essential to ensure that a safe and efficient satellitebased navigation service is provided to all users.

With the development of satellite-based CNS/ATM technologies, Civil Aviation Authorities (CAAs) around the world face the challenge that the next generation
ATC and ATM systems are likely to be increasingly dependent upon the operation of GNSS systems such as GPS that are out of their operational control. While the benefits of GNSS have been recognized by ICAO, the solution to meet the challenge of dependence without control has not been finalized.

ICAO Document A32-19: The Charter on the Rights and Obligations of States Relating to GNSS Services was adopted by the 32nd Session of the Assembly, in October 1998. It states:

- Section V3-2: Every State and aircraft of all States shall have access, on a nondiscriminatory basis under uniform conditions, to the use of GNSS services, including regional augmentation systems for aeronautical use within the area of coverage of such systems.

- $\quad$ Section V3-4: Every State providing GNSS services, including signals, or under whose jurisdiction such services are provided, shall ensure the continuity, availability, integrity, accuracy and reliability of such services, including effective arrangements to minimize the operational impact of system malfunctions or failure, and to achieve expeditious service recovery.

In addition, the unique characteristics of the ionosphere in the Southern Latitudes requires the ability to monitor GNSS signals in real time and report effects on the levels of service throughout the National Airspace.

Satellite navigation systems have generated a challenge to predicting availability of service. The impact of a satellite outage is not intuitive due to the dynamics of the constellation. A real-time performance monitoring and prediction capability is needed as an essential tool to 
ensure the safe operation of all satellite navigation systems authorized to operate in the any National Airspace. This capability is required in order to achieve the following minimum requirements for GNSS safety and integrity:

- Continuous real time status monitoring of GNSS for airport landing applications (ICAO Annex 10, Chapter 2.8)

- Continuous real time data storage of GNSS performance for liability and accident investigations (ICAO Annex 10, Chapter 2.4.3)

- $\quad$ Provide Notice to Airmen (NOTAM) to users when GNSS cannot be used for specific safety applications (ICAO Annex 10, Chapter 3.7.6)

- Verification that the local interference environment is not harmful to GNSS users (ICAO Annex 10, Chapter 3.7.4)

There currently exists no capability to generate real-time, graphical aeronautical information for space-based navigation aids or the associated augmentation systems. A GNSS Performance Monitoring System must be responsible for ensuring satellite-based systems, including GPS, Galileo, WAAS, EGNOS, MSAS, LAAS, and future GNSS, SBAS and GBAS services, provide a continuous, safe, and reliable signal-in-space (SIS).

Brazil's Department of Air Space Control (DECEA) in cooperation with the US Volpe Transportation Center and Innovative Solutions International (ISI), Inc., has embarked on a program to develop and deploy the first real time GNSS performance monitoring capability for operational use. The system will be able to monitor the operational status of a GNSS system in real time, as well as predict its future performance as well as possible. The functions of the GNSS Performance Monitoring System (GPMS) are:

- Monitor the performance of GNSS or SBAS signals in real time, including the ionosphere

- Provide status as to the availability of the different levels of approach capabilities such as LNAV/VNAV, LPV, or non-precision approaches, and predict availability of those services into the future

- Generate aeronautical information for use in development of NOTAMS

- Provide a database of performance for analysis and to reproduce past performances for investigations, if needed

\section{TECHNICAL DESCRIPTION}

Traditional NOTAMS are typically site and service specific. Satellite-navigation-specific NOTAMS present unique challenges to the NOTAM system, in that an outage can affect large geographic areas and encompass multiple airports with different levels of service. The following is a brief description of the current distribution of NOTAMs: GPS satellite outages are sent from the GPS Master Control Station at Schriever AFB to the U.S. NOTAM Office a minimum of 48 hour notification for scheduled GPS space vehicle (SV) outages (1 week notice generally given). Unscheduled outages are generally distributed within 30 minutes. GPS SV outages are distributed domestically under the KGPS Identifier and internationally under the KNMH Identifier. Countries not receiving GPS SV NOTAMs submit requests for KNMH NOTAMs to U.S. NOTAM Office and provide AFTN address.

The impact to flight operations is not readily apparent. In order to support the operational use of GNSS the system will have the following capability:

1) Independent Monitoring - The system will have the capability to assess near term ( $<2$ hours) and longterm performance of all monitored GNSS systems. Near term assessment will allow the operator to provide real time information to Air Traffic Control Facilities as to availability and continuity parameters for the system and the impact on approach availability. Output will be a screen that displays current system performance with a limited amount of trend information.

2) Airspace and augmentation system prediction and planning - The system will have the capability to develop system performance predictions based on past performance, current performance, ionospheric data, system equipment status, and terrain. The operator will have the capability to generate reports with some level of associated analysis.

3) Notice to Airmen (NOTAMS) - The system will have the capability to generate information in real time pertaining to system performance and its impact on the airspace to the appropriate national agency responsible for generating the NOTAMS (text and graphical).

4) Public dissemination of performance information The system will have a web site available to the public at which the users can access system performance information to plan flights, download data, or provide feedback to the users on interference/system performance. 
5) Data Collection and Archiving - The system will have the capability to access the archived data for accident investigation and formulate reports.

The figure below depicts a high-level system overview of the Brazilian GPMS. The Brazilian Test Bed (BTB) has been integrated into the GPMS as a subsystem. To enhance the signal-in-space (SIS) monitoring coverage, a series of GNSS Local Monitors (LM) will be installed at selected airports geographically dispersed from existing BTB monitoring station locations. These monitors provide additional data collection to support the GPMS to monitor GNSS signal performance over the Brazil national airspace. The end state capability of the GPMS will eventually include data from all GBAS, SBAS, and GNSS Local Montor installations.

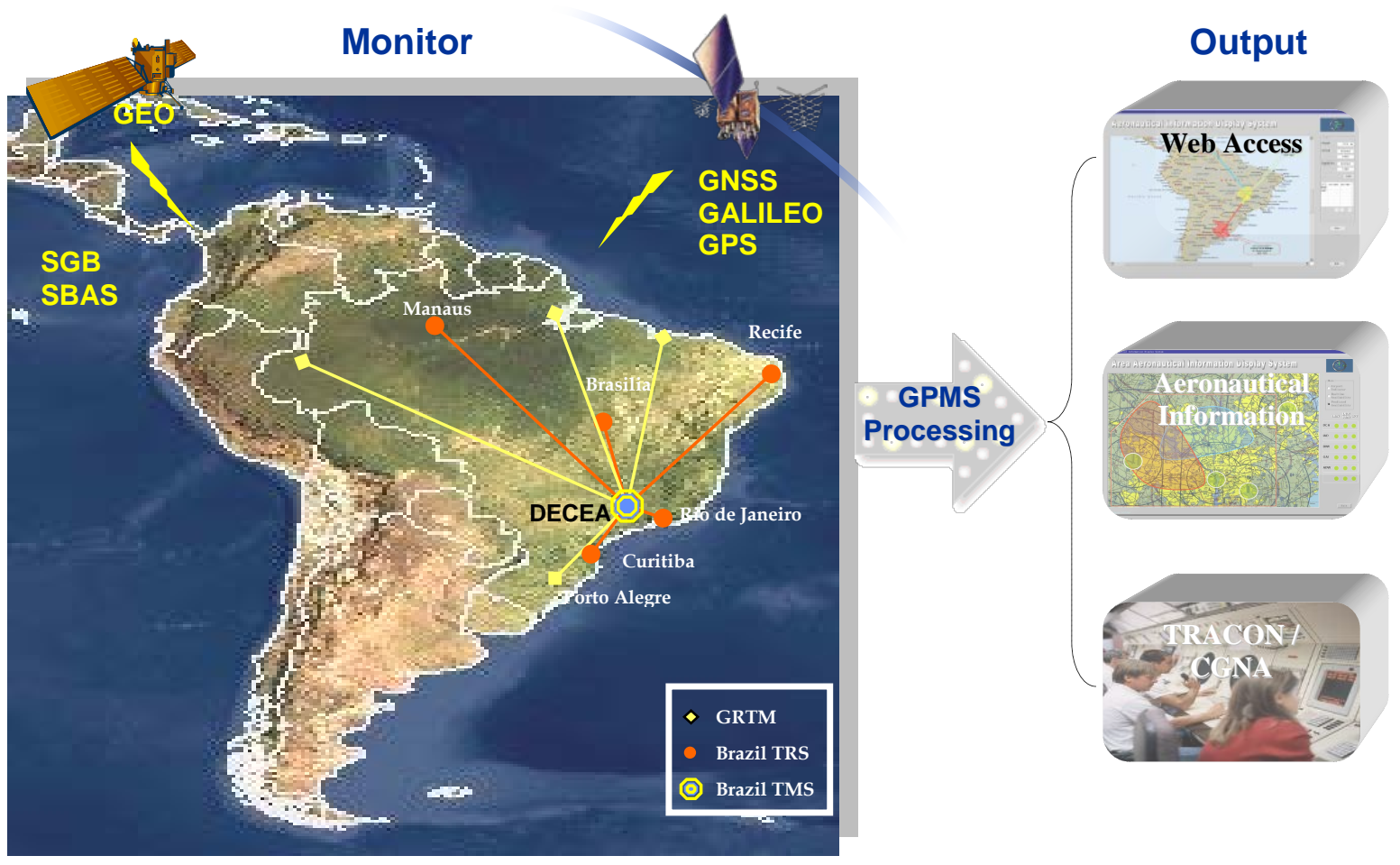

Figure1. Brazilian GPMS Description

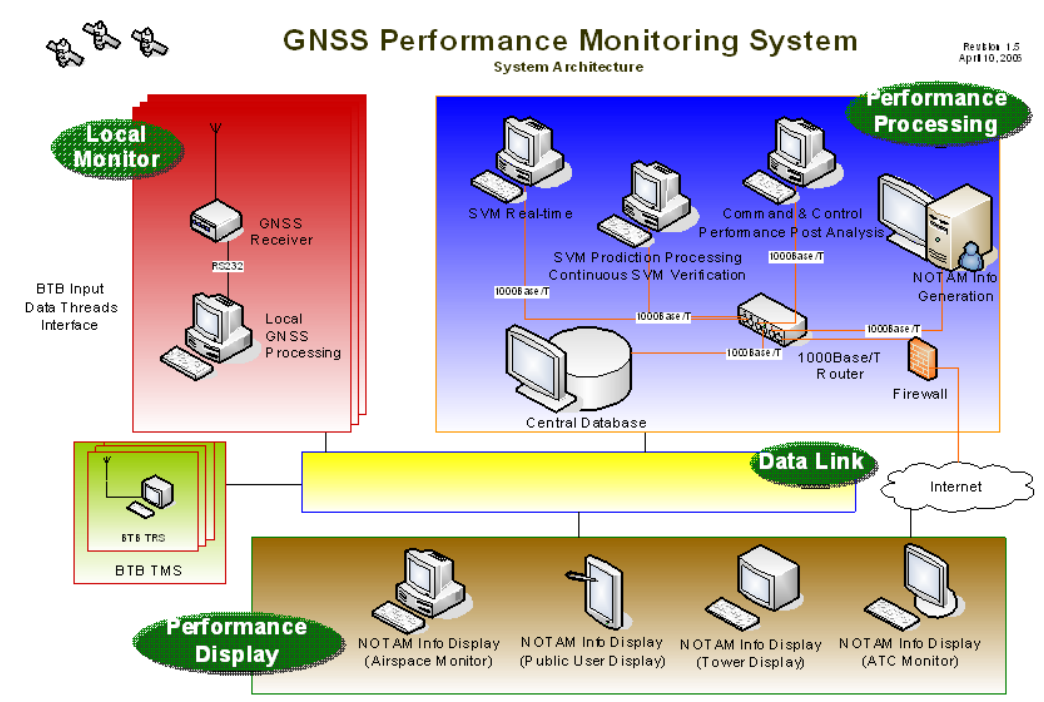

Figure 2. Brazilian GPMS Architecture Diagram 


\section{GPMS ARCHITECTURE}

Figure 2 shows the Brazil GPMS system architecture diagram that depicts the functional decomposition of the system into major architectural elements as GPMS functional modules, together with the GPMS external interfaces and internal functional connections.

The baseline GPMS system is composed of the following major subsystems and architectural elements as follows:

Brazil Test Bed (BTB) Subsystem and Local GNSS Monitor Subsystem

- GNSS Receiver Module

- Local GNSS Monitor Processor Module

- BTB Data Acquisition Module

GPMS Performance Processing Subsystem

- SVM Real Time Module

- SVM Prediction Module

- Central Database Module

- Continuous SVM Validation Module

- NOTAM Information Generation Module

- Performance Post Analysis Module

Data Link Subsystem

- Data and Control Communication Implementation

GPMS Performance Display Subsystem

- Airspace Monitor Module

- Public User Display Module

- Tower Display Module

- $\quad$ ATC Monitor Module

The Brazil Test Bed (BTB) is assumed operational and has been integrated into the GPMS as a subsystem. The GPS signal in space (SIS) data collected and processed by BTB will be forwarded to GPMS's central database and used by other GPMS subsystems and modules, such as the SVM Real Time Processing Module and the SVM Prediction Module for further processing. To enhance the SIS monitoring coverage, the Local GNSS Monitor Subsystems (LMs) will be installed at select airports that are geographically dispersed from existing BTB monitoring station locations.

These local GNSS monitor subsystems provide additional data collection to support the GPMS to monitor GNSS signal performance over the Brazil national airspace. In addition, each local monitor will conduct independent GNSS performance monitoring and predict the near term operational performance of the GNSS of concern for the airport where it is installed and operated.

\section{BTB and Local Monitors}

The BTB is already installed and operational. The BTB is a SBAS test bed used by DECEA to conduct analysis and flight trials concerning SBAS performance. The Brazil GNSS Performance Monitoring Network will use BTB as a component to perform the following functions:

a) Collect GNSS SIS data;

b) Perform GNSS SIS navigation data correction and integrity processing;

c) Forward data collected in a) and generated in b) above to the Main Database Subsystem.

The interface between BTB and the Central Database module is implemented through the multicast mechanism implemented in BTB master station. Key data elements include raw GPS measurements data at each BTB monitoring station plus GPS differential correction and integrity data generated by the BTB master station. The logical interface between BTB and the Central Database module is implemented through the Data Link Subsystem and is a one-way data flow.

GPMS local monitor stations will be installed at multiple airport locations. The local monitor station including its GUI performs the following functions:

a) Collect GNSS SIS data (at the local monitor station location in addition to BTB sites);

b) Perform GNSS SIS navigation data integrity processing (at the local monitor station site);

c) Perform GNSS SIS performance prediction (at the local monitor station site for 60 minutes time period);

d) Display GNSS SIS real time performance monitoring and prediction results;

e) Forward GNSS SIS data collected in a) and generated in b) and c) above to the Central Database module for further processing by the SVM Real Time module.

The interface between local monitor and the Central Database is implemented through the Data Link Subsystem and is a one-way flow for application data and a two-way flow for command and control. Key data items include GPS real time ephemeris data, GPS raw measurements at the local monitor station antenna location, and GPS integrity data as determined by the local monitor processor. This logical interface is stressed because the real time SVM is the primary use of local monitor GNSS SIS measurements data. 


\section{SVM Real Time and Prediction Processing}

With wide-area differential GPS systems such as WAAS and EGNOS, a service volume model (SVM) capable of generating real time information is an essential assessment tool in order to develop the capability to generate information useful to daily flying operations. The operational service volume model that predicts the performance of RAIM (TSO C129) and SBAS (TSO C145/146) receiver equipment will be used.

The Performance Processing Subsystem consists of CAC (Command and Control), CDB (Central Database), SRT (SVM Real-time), SPP (SVM Prediction Processing), CSV (Continuous SVM Validation), and NIG (NOTAM Information Generation). The PP accepts data from up to 5 BTB TRSs (Testbed Reference Station) and up to 5 Local Monitoring Subsystem (LM) and perform the functions of data validation check, satellite orbit computation, RAIM computation and service availability determination, validation, and NOTAM information generation.

By using the data from both BTB TRSs and LMs, the PP determines wide area and local area RAIM availability in both real-time and perdition modules. The PP collects the data received from all BTB TRSs and LMs in real time and processes it to perform these preceding functions.

A set of NOTAM information is formed based on the results from the SRT and SPP and forward to CDB.

The Performance Processing embodies processing equipment necessary to perform the PP functions, equipment to interface the PP with the Data Link Subsystem (DL), and Human-machine interface equipment. The SRT performs the real time service volume model functions; it is hosted on one CP (Computer Processor). The SPP performs the service volume model prediction functions; it is hosted on another CP.

The Performance Processing module also has a data recording and archiving capability. Locally generated data and data received from the BTB TRSs and LMs are recorded in the central database.

The Performance Processing module performs the following functions to perform its role in generating the NOTAM information for various user requirements:

a) Compute GPS/GEO satellite orbits,

b) Compute the Receiver Autonomous Integrity Monitoring (RAIM), both FD and FDE, in real time,

c) Compute the Receiver Autonomous Integrity
Monitoring (RAIM), both FD and FDE, for long term prediction,

d) Record and archive the input data and the calculated data,

e) Validate calculated data for both real time and prediction SVM,

f) Monitor other GNSS performance,

g) Generate NOTAM information messages and graphics.

The heart of SVM Prediction Processing module is the GNSS performance future prediction function. The use of satellite navigation systems has generated a challenge to predicting the availability of service. The impact of a satellite outage is not intuitive due to the dynamics of the constellation; therefore a predictive model is required to determine the affected area.

The SVM Prediction Processing module will support the following GPS receiver types and combinations with selection performed by the system operator using the offline preference settings in the Graphical User Interface (GUI).

The software will support the following algorithms and inputs:

- TSO C129a RAIM prediction

- $\quad$ TSO C145/146a RAIM prediction

- Option of including of barometric altimeter aiding

- Option of including of WAAS geostationary satellites (applies to TSO C145/146a receiver only)

- Option to include PRN codes and longitudes for new geostationary satellites

- Option to vary receiver mask angle setting

The SVM will run once per day at a scheduled time to support the non-real time analyses. The SVM shall recalculate at least once every 24 hours for the following 48-hour period. If an outage or change in predicted service is declared in a GPS or Geostationary SV NOTAM or the airfield database is updated, the RAIM algorithm is immediately reprocessed.

The output of all of the GPS RAIM prediction algorithms is the Horizontal Protection Level (HPL). In addition, the SVM will provide predicted SV visibility and DOP values. In the future if vertical guidance is supported, a VPL will be output.

The SVM Prediction Processing shall occur at 1-degree latitude/longitude grid points over Brazilian airspace. The defined area of interest will be located in the offline preference settings. 
A GPS RAIM outage is defined as a period of time when a GPS receiver is expected to output an HPL value exceeding a threshold value defined in the offline preference setting. The Horizontal Alert Limit (HAL) or threshold for non-precision approach (NPA) is $0.3 \mathrm{nmi}$ $(555.6 \mathrm{~m})$. Wide area RAIM availability for en route and terminal operations will be finalized in GPMS SSDD.

The information transmitted will include the expected start and end time of each outage for each cell or groups of cells with a spatial resolution of 1 minute. The SVM processing shall occur for the database of airfields defined by DECEA. A GPS RAIM outage is defined as a period of time when a GPS receiver is expected to output an HPL value exceeding a threshold value defined in the offline preference setting. The Horizontal Alert Limit (HAL) or threshold for non-precision approach (NPA) is $0.3 \mathrm{nmi}(555.6 \mathrm{~m})$.

The Central Database performs the following functions:
a) Receive data through Data Link Subsystem from the BTB Subsystem;
b) Receive data through Data Link Subsystem from the Local GNSS Monitor Subsystems;
c) Receive data (through Data Link Subsystem) from SVM Real Time module;
d) Receive data (through Data Link Subsystem) from SVM Prediction Processing module;
e) Organize and store data received in a) through d) for maximum data retrieval flexibility and efficiency;
f) Receive and respond to database requests from SVM Real Time module;
g) Receive and respond to database requests from SVM Prediction Processing module;
h) Receive and respond to database requests from Continuous SVM Validation module; i) Receive and respond to database requests from
NOTAM Information Generation module
j) Respond to database requests from various NOTAM Information Display modules for use by GPMS end users.

The NOTAM Information Generation module will process GNSS operational performance status and predicted performance generated by the SVM Real Time and SVM Prediction Processing modules obtained through the central database to generate NOTAM information that is in compliant with ICAO NOTAM format for dissemination and NOTAM information display.

The NOTAM Information Generation will support the following functions:
- Text-based NOTAM generation and dissemination

- Graphical-based NOTAM display

- Web-based NOTAM Publishing

The Text-based NOTAM Generator module shall provide the following functions:

a) Acquire data, generated by the SVM Prediction module, from the Central Database;

b) Extract information pertinent to NOTAMs, from data acquired above;

c) Generate NOTAM data in ICAO-compliant text format;

d) Based on the SISNOTAM (NOTAM Management System) NOTAM data will be submitted to an external interface for further national distribution;

e) Process NOTAM acknowledgement messages from SISNOTAM for tracking assigned NOTAM Numbers, to facilitate cancellation of existing NOTAMs if required.

The Graphical-based aeronautical information module shall provide the following functions:

a) Acquire data, generated by both the SVM Prediction Processing and SVM Real Time modules, from the Central Database;

b) Extract information pertinent to NOTAMs, from data acquired above;

c) Generate and Display NOTAM information in graphical form.

The Web-based NOTAM Publishing module shall provide the following functions:

a) Receive from the Web and process "information requests" for NOTAM information (text and/or graphical);

b) Request NOTAM information (text and/or graphical) through the Central Database from the NOTAM Generator Subsystem and/or the graphical aeronautical information;

c) Release NOTAM information (text and/or graphical) to the Web requester.

\section{Data Link Subsystem}

The Data Link Subsystem performs the following functions:

a) Transport data between BTB and Central Database;

b) Transport data between local monitor station and Central Database. 
It is assumed that all interfacing equipment will be located at the same sites, including the BTB master station, the GPMS local monitor subsystem, the SVM Real Time module, and the SVM Prediction module. The Data Link Subsystem will use third party telecommunications infrastructure to develop WAN connectivity when multiple local monitor stations that are installed at geographically dispersed locations.

\section{Performance Display Subsystem}

The GPMS Performance Display Subsystem is designed to consist of four end-user display modules:

- Tower Display

- ATC Area Monitor

- $\quad$ Public User Display

- Airspace Monitor

\section{Tower Display}

The Tower Display module will display GNSS operational information generated by the GPMS local monitor stations installed at airports and ATC towers. The primary intended users of the Tower Display module are ATC controllers at ATC towers responsible for providing terminal and aircraft approach and landing control services.

The two key elements of the of Tower Display module are:

- $\quad$ GNSS SIS performance including accuracy and integrity at the airports; and

- $\quad$ Predicted SIS performance (30 minutes - 2 hours ahead) at the airports.

The Tower Display design must be simple and straightforward taking into consideration of the heavy workloads and information overloads to ATC controllers at the towers.

\section{ATC Area Monitor}

The ATC Area Monitor module will display GNSS operation information generated by the GPMS over the area of ATC area of interest, such as one or multiple ATC sectors. The primary intended users of the ATC Area Monitor are ATC sector and area controllers.

The key elements of the ATC Area Monitor module are:

- Instantaneous GNSS availability for the ATC sector;

- $\quad$ Predicted GNSS availability (6 hours) for the sector.
The primary purpose of this display is for the sector controller to manage his airspace and coordinate with adjacent sectors.

\section{Public User Display}

The Public User Display module will display GNSS operational status and predicted performance generated by GPMS over the entire Brazilian airspace. The primary intended users of the Public User Display module are pilots while conducting flight planning. The module will implemented over the Internet and deployed primarily at the Brazilian air field operations offices.

The key elements included in the Public User Display module are:

- $\quad$ GNSS NOTAMs - Regional and Airport

- $\quad$ GNSS Service Availability (RAIM)

- Predicted

- Instantaneous

The user will be allowed to insert the intended flight information, such as departure time and airport, routes, destination, and arrival time. The module to provide the user with text and graphical NOTAMS along each intended flight route.

\section{Airspace Monitor}

The Airspace Monitor module will be very similar to SVM Real Time module GUI and it will display GNSS operational status and predicted performance generated by GPMS over the entire Brazilian airspace. The primary intended users of the Airspace Monitor module are airspace managers and planners, SBAS designers, and GNSS researchers.

Key elements included in the Airspace Monitor module are:

- GNSS instantaneous NOTAM information

- $\quad$ Predicted NOTAM information (graphical and text)

- $\quad$ System performance information using the tool to conduct "what if" scenarios

- RAIM availability

- Archiving

As previously stated, traditional NOTAMS are typically site and service specific. Satellite-navigation-specific NOTAMS present unique challenges to the NOTAM system, in that an outage can affect large geographic areas and encompass multiple airports with different levels of service. Flight Service Station and/or Air Traffic personnel require the capability to monitor and predict short-term fluctuations in the satellite navigation 
service volume on a real-time basis in order to safely support flight operations in the national airspace system. The GPMS will integrate a real-time SVM and a graphical display capability to generate accurate realtime local and area NOTAM information that complies with the ICAO text standard. The real time performance monitoring network will have the capability to assess near-term (within 2 hours) and long-term (greater than 2 hours) performance of all monitored satellite navigation systems. Near-term assessment must allow the operator to provide real-time information to Air Traffic Control Facilities about the availability and continuity parameters for the system and the impact of these parameters on approach availability

\section{PROJECT ORGANIZATION}

The project is divided into two phases as outlined below;

PHASE I: (Requirements Definition, Prototype)

This first phase consists of the development/delivery of the system modules to include the Regional Performance Monitoring, the Operational Service Volume Model, the generation and dissemination of aeronautical information.

- Initial Procurement - Hardware / Software

- Data and Comm Links to support processing/storage of input (including 5 test bed stations and one ATC)

- Database (Schema Definition, Prototype of short/long term storage and retrieval)

- Initial Development / Testing of Real-Time and Predictive "SVM Core" modules

- Regional Performance Monitoring (Prototype)

- Aeronautical Info Generation \& Dissemination (Conceptual Demo)

PHASE II: (FSD, Operational Readiness, System Validation)

The second phase provides for the operation and maintenance assistance required by DECEA to operate the system at the desired capability.

- Additional Procurement for Commissioning

- Development / Testing of Interactive Graphical Monitoring (with prediction and planning capability)

- $\quad$ Development / Testing of Graphical Output Generation and Dissemination (C/S and B/S based Dissemination)

- V\&V - SVM Core Modules, Database, Comm Links (Internal and External)

- End-to-End Testing of Distribution (NOTAMs, Web, Graphics)

- $\quad$ Training / Deployment
Both Phase I and Phase II of GPMS are expected to be complete by December 2006.

\section{Summary}

GPMS will provide nations the capability to manage, monitor, and control all GNSS signals within a National Airspace System to support the transition to satellite based CNS/ATM and meet ICAO requirements.

The capability to integrate, monitor, and assess the operational performance of GNSS systems is essential to ensure that a safe and efficient satellite-based navigation service is provided to all users.

DECEA is well on its way to deployment of a system will be able to monitor the operational status of a GNSS system in real time, as well as predict its future performance as well as possible. It is expected to be fully operational by the end of 2006. 